\title{
Nuevos registros y observaciones de algunas especies de Elaphoglossum (Elaphoglossaceae) del Perú
}

\section{New records and observations of some species of Elaphoglossum (Elaphoglossaceae) of Peru}

\author{
Leopoldo F. Mellado y Blanca León
}

Museo de Historia Natural, Universidad Nacional Mayor de San Marcos, Av. Arenales 1256, Apartado 14-0434, Lima-14, Perú. Email Leopoldo F. Mellado: I.franco.mellado.n@gmail.com Email Blanca León: blanca.leon@mail.utexas.edu

Presentado: 10/07/2006 Aceptado: $\quad 14 / 02 / 2007$

\section{Resumen}

Elaphoglossum cardioglossum Mickel y Elaphoglossum molle (Sodiro) C. Chr. son reportados para la flora peruana, de colecciones realizadas en Cusco y Pasco. Por primera vez es descrito material fértil de Elaphoglossum alipes Mickel, Elaphoglossum atropunctatum Mickel, Elaphoglossum cardioglossum Mickel y Elaphoglossum haynaldii (Sodiro) I. Losch. También se actualizan sus distribuciones.

\section{Palabras Claves: Elaphoglossaceae, Elaphoglossum, Pteridophytas, helechos, Perú. \\ Abstract}

Elaphoglossum cardioglossum Mickel and Elaphoglossum molle (Sodiro) C. Chr. are reported for the first time for the Peruvian flora, from collections made in Cusco and Pasco. We also describe for the first time fertile material of Elaphoglossum alipes Mickel, Elaphoglossum atropunctatum Mickel, Elaphoglossum cardioglossum Mickel and Elaphoglossum haynaldii (Sodiro) I. Losh, and we update their current distributions.

Keywords: Elaphoglossaceae, Elaphoglossum, pteridophytes, ferns, Peru.

\section{Introducción}

Dentro de los helechos, el género Elaphoglossum es uno de los más complejos y taxonómicamente dificultoso. También es uno de los más grandes en los Andes (Smith et al., 2005). Está conformado por más de 600 especies (Mickel, 1985; 1990), de las cuales cerca de 450 habitan en el Neotrópico (Moran, 1995; Skog et al., 2004)

A pesar de ser un grupo de una gran dificultad para la determinación de especies, es uno de los géneros más fáciles de reconocer, por sus hojas simples con soros acrosticoides cubriendo toda el envés de la fronda, generalmente con venas libres. En su mayoría son plantas epífitas (Mickel, 1980; 1991; Mickel \& Atehortúa, 1980), aunque pueden también ser terrestres o epipétricas (Mickel, 1991). La morfología y el indumento foliar proveen la mayoría de los carácteres taxonómicos para el reconocimiento de especies, mientras que las estructuras fértiles contribuyen a reconocer grupos afines.

Los últimos reportes para el Perú, mencionan a Elaphoglossum con un total de 137 especies (Mickel, 1991; Rojas Alvarado, 2002; Smith et al., 2005). De esas especies 15 fueron descritas de ejemplares estériles, entre ellas las tres especies que aquí se discuten, y de las cuales dos de ellas son endémicas del Perú (Mickel, 1991).

\section{Materiales y métodos}

El material estudiado se halla depositado en el Herbario San Marcos (USM) y generalmente corresponden a colectas realizadas durante proyectos de investigación de los autores. El primer autor trabajó en el Parque Nacional Yanachaga Chemillén, durante la investigación de la taxonomía y ecología del género Elaphoglossum, y el segundo autor en el Parque Nacional Manu y en el Parque Nacional Río Abiseo.

Para la determinación botánica se utilizaron claves de identificación y bibliografía especializada, también se uso la base fotográfica y las notas inéditas de Elaphoglossum de Bolivia de M. Kessler et al. (2004).

\section{Resultados}

Dos especies de Elaphoglossum, E. cardioglossum Mickel y Elaphoglossum molle (Sodiro) C. Chr., son registros nuevos para la flora del Perú. Se describe muestras fértiles de cuatro especies: Elaphoglossum alipes Mickel, Elaphoglossum atropunctatum Mickel, Elaphoglossum cardioglossum Mickel y Elaphoglossum haynaldii (Sodiro) I. Losch.

A continuación se presentan las especies en orden alfabético.

\section{Elaphoglossum alipes Mickel}

Fieldiana, Bot., n.s. 27: 122. 1991.

Tipo: Perú, Ucayali (como Loreto), along Rio Aguetia (Aguaytía) above mouth of Quebrada Yurac-Yacu, 2 oct 1972, Croat 20857 (holotipo, UC!).

Rizoma cortamente repente, erguido, $3 \mathrm{~mm}$ de diámetro; escamas del rizoma lineares, de negras a marrón-anaranjadas, lustrosas, enteras, $1-2(3) \mathrm{mm}$, muy, algunas veces piliformes; filopodio ausente; hojas fasciculadas, $10-37 \times 1,5-3,5 \mathrm{~cm}$; pecíolo ausente; lámina oblanceolada a elíptica, cuando fresca carnosa, secas algo papiráceas, ápice agudo, base largamente decurrente, secas marrón claro, con manchas regulares de cera blanca entre las venas secundarias; nervaduras conspicuas, $2-2,5 \mathrm{~mm}$ espaciadas, en un ángulo de $65-75^{\circ}$ con respecto a la costa, libres; hidatodos evidentes; escamas de la lámina subuladas, enteras, $2-3 \mathrm{~mm}$, mayormente distribuidas a lo largo del borde de la lámina; escamas del haz iguales a las del envés; hoja fértil más corta del largo de la hoja estéril; pecíolo $1 / 2-2 / 3$ de la longitud de la hoja fértil, escamas del pecíolo de la hoja fértil subuladas, enteras, castañas, $2 \mathrm{~mm}$ de largo; lámina fértil elíptica, ápice agudo, base decurrente, borde entero; escamas interesporangiales subuladas, enteras, castañas, $1-2 \mathrm{~mm}$ de largo, escamas de la costa ausentes.

Hierba, epífita. 380—510 m de altitud. En bosque amazónico. 
Este especie epífita era sólo conocida del tipo, proveniente de una localidad en la cuenca del Aguaytía, de altitud desconocida. Con este trabajo se reporta su presencia por debajo de los $550 \mathrm{~m}$. Se reporta por primera vez datos de las hojas fértiles, las cuales se caracterizan por su menor tamaño que las estériles.

Distribución: Anteriormente endémica de Ucayali y ahora reportada para Pasco.

Material examinado: PERÚ: Dpto. Pasco. Prov. Oxapampa. Dist. Palcazu: P. N. Yanachaga Chemillén, sector Paujil, colecta camino a colpa, bosque primario; selectivamente intervenido, 380 m, 26 feb 2005, Mellado 2843 (AMAZ, HUT, MO, USM, Herbario Selva Central); San Pedro de Pichanaz: Sector Azulis, Reserva Comunal Yanesha, bosque de Arena Blanca, 510 m, 26 feb 2004, Mellado, Monteagudo \& Rodríguez 0795 (USM).

\section{Elaphoglossum atropunctatum Mickel}

Fieldiana, Bot., n.s. 27: 124. 1991.

Tipo: Perú, Pasco, Oxapampa, 2-4 km N of Mallampampa, D.N. Smith \& Canne 5835 (holotipo MO, isotipo USM!)

Rizoma compacto, 3-5 mm de diámetro; escamas del rizoma linear-lanceoladas, marrones, de la mitad de la escama a la base más claras, ligeramente dentadas, $7-8 \times 1-0,9 \mathrm{~mm}$; filopodio presente, $5-11 \mathrm{~cm}$ de largo, generalmente cubierto por escamas; hojas 1,5-5 mm distanciadas, 20,5-37 x 2,5-5,1 cm; pecíolo $1 / 2$ - 2/3 del largo de la hoja estéril; escamas del pecíolo ovado lanceoladas, erosas o cortamente ciliadas, $4-5 \times 1,5-2,5 \mathrm{~mm}$, castaño-blanquecinas con el centro negro, acompañadas por escamas más pequeñas ovado lanceoladas cilio denticuladas y con el centro negro; lámina de ovada a angostamente elíptica, cartácea, ápice redondeado, base anchamente cuneada, borde entero; nervaduras inconspicuas, 1,5 $\mathrm{mm}$ espaciadas, en un ángulo de $60-70^{\circ}$ con respecto a la costa, libres; hidatodos ausentes; escamas de la lámina de ovadas a lanceoladas, con cortos dientes piliformes, 1 x 0,5 mm, negras (castañas), entre estas escamas más pequeñas redondeadas, peltadas, con cortos dientes piliformes, castañas; escamas del haz de ovado a lanceoladas, cilio denticuladas, castaño-blanquecinas, $1-1,5 \mathrm{~mm} \times 0,6-0,9 \mathrm{~mm}$, de esparcidas a abundantes, algunas veces la lámina glabrescente; hoja fértil ligeramente más larga que la hoja estéril; pecíolo 3/5 de la longitud de la hoja fértil; lámina fértil linear-elíptica, ápice obtusa a redondeada, base cuneada, borde entero; escamas interesporangiales ausentes, algunas escamas pequeñas ovado-lanceoladas erosas denticuladas adpresas cobre la costa.

Hierba, epífita. 2070-3500 m de altitud. En bosque montanos hasta bosque montano muy húmedo.

Esta especie era conocida de ejemplares estériles procedentes del centro del país. Aquí se describe por primera vez la hoja fértil, la cual alcanza un largo similar al de la hoja estéril. Esta especie puede crecer como una planta epífita o sobre abundante materia orgánica en el sotobosque de bosques muy húmedos. Su amplitud altitudinal supera los $1500 \mathrm{~m}$.

Distribución: Endémica en los departamentos de Huánuco, Pasco y Junín.

Material examinado: PERÚ: Dpto. Pasco. Prov. Oxapampa. Chontabamba: Sector La Suiza Vieja, Zona de Amortiguamiento del P. N. Yanachaga Chemillén, 2195 m, 07 ene 2005, Mellado, Arias \& Francis 2347a (HUT, MO, NY, USM, Herbario Selva
Central); Huancabamba: P. N. Yanachaga Chemillén. Quebrada Yanachaga, bosque primario, 2250 m, 13 jun 2003, Vásquez et al. 28206 (MO, USM!); 2070 m, 14 set 2004, Mellado 1715 (MO, USM, Herbario Selva Central); 2100 m, 16 set 2004, Mellado 1764 (USM); 2220 m, 17 oct 2004, Mellado, Perea, Becerra \& Mateo 1986 (USM); 2262 m, 18 set 2004, Mellado \& Becerra 1784 (USM); Oxapampa: P. N. Yanachaga Chemillén, Sector San Alberto, 2200 m, 20 ene 2003, Vásquez, Rojas, Monteagudo, Ortiz, Mateo 27857 (MO, USM); Oxapampa: 2200—2400 m, 22 ene 1984, Smith 5835 (USM!); Oxapampa: Norte de Mallampampa, 2200-2400 m, 22 ene 84, Smith \& Canne 5861 (MO, USM!); Oxapampa: Sector Quebrada San Alberto P. N. Yanachaga Chemillén, colecta siguiendo el camino al abra Esperanza, $2630 \mathrm{~m}$, 24 oct 2004, Mellado \& Becerra 2112 (USM); Oxapampa: Abra Villa Rica bosque sobre Arenisca blanca, 2400 m, 25 ago 2004, Mellado 1618 (USM); 1619 (MO, USM); 1637 (MO, USM).

\section{Elaphoglossum cardioglossum Micke}

Brittonia 37(3), 1985 pp. 261-278

Tipo: Bolivia, Departamento La Paz. Provincia Nor-Yungas, "Coroico 39 km hacia La Paz, 3100 m”, Beck 587 (holotipo: NY)

Hoja fértil más larga que la hoja estéril; pecíolo aprox. 4/5 de la longitud de la hoja fértil; lámina fértil igual a la lámina estéril aunque más angosta $(2,2 \mathrm{~cm})$, base cordada; escamas interesporangiales presentes, lanceoladas, marrón oscuras, enteras a ligeramente dentadas de $0,5 \mathrm{~mm}$ de longitud, muy esparcidas; envés con escamas de $1-1,5 \mathrm{~mm}$ de longitud esparcidas.

Elaphoglossum cardioglossum Mickel fue descrita en 1985 de materia estéril para el departamento de la Paz en Bolivia y se caracteriza por presentar hojas prolíferas. En este trabajo se amplia su distribución sobre los $3300 \mathrm{~m}$ de altitud y se describe por primera vez su hoja fértil, la cual es más larga y angosta que la hoja estéril.

Esta especie está cercanamente relacionada con Elaphoglossum pascoense R. M. Tryon. Elaphoglossum cardioglossum se reconoce por su rizoma cortamente repente y sus hojas ovadas de 5-6,5 $\mathrm{cm}$ de ancho, mientras que $E$. pascoense presenta rizoma largamente repente y sus hojas van desde $6,5 \mathrm{a} 12 \mathrm{~cm}$ de ancho.

Distribución: Anteriormente especie endémica de Bolivia, se extiende su distribución al Perú en el departamento del Cusco.

Material examinado: Perú: Cusco, Paucartambo P. N. Manu, en la Trocha Ericson, 3220-3320 m de altitud, León 2758 (USM).

\section{Elaphoglossum haynaldii (Sodiro) I. Losh}

Mitt. Bot. Staatssamml. Munchen 1: 21. 1950

Acrostichum haynaldii Sodiro, Recens. Crypt. Vasc. Quit. 80. 1883.

Tipo: Ecuador: Pichincha Sodiro s.n. (holotipo: no localizado).

Rizoma cortamente repente, ascendente, glutinoso, $5 \mathrm{~mm}$ de diámetro; escamas del rizoma lanceoladas, marrón oscuro, enteras a irregularmente dentadas, $5 \mathrm{~mm}$ de largo; filopodio ausente; hojas fasciculadas, $40 \times 1,3-2,4 \mathrm{~cm}$; pecíolo $1 / 4$ del largo de la hoja estéril; escamas del pecíolo subuladas, enteras, 4-6 mm de largo, rojizas, muy esparcidas; lámina linear-elíptica, cartácea, ápice acuminado a ausente, base cuneada, borde entero; nervaduras inconspicuas, $1,5-2 \mathrm{~mm}$ espaciadas, en un ángulo de $60^{\circ}$ con respecto a la costa, libres; hidatodos evidentes; escamas de 
la lámina subuladas, enteras, 4-6 $\mathrm{mm}$ de largo, regularmente distribuidas, algunas veces tricomas glandulares erguidos sobre la lámina; escamas del haz iguales a las del envés; hoja fértil igual o ligeramente más corta del largo de la hoja estéril; pecíolo $1 / 4-1 / 5$ de la longitud de la hoja fértil; lámina fértil igual a la lámina estéril aun que más angosta $(9-1,2 \mathrm{~cm})$, ápice agudo a ligeramente mucronato o ausente; escamas interesporangiales ausentes; escamas subuladas iguales a las de la hoja estéril sobre la costa y la haz de la hoja fértil.

Hierba, epífita facultativa, péndula. 1600 -2000 m de altitud. En bosque montano perturbado.

Esta es una las 15 especies descritas por Mickel en 1991 para la flora peruana, sobre la base de material estéril. Se amplia su distribución altitudinal desde los 1600 hasta los $2800 \mathrm{~m}$ de altitud y se describe la hoja fértil, la cual se caracteriza por presentar escamas subuladas a lo largo de la costa.

Distribución: Anteriormente conocida en el Perú en los departamentos de Ayacucho y Cusco, Se extiende su distribución para los departamentos de Piura, San Martín y Pasco.

Ecuador, Perú y Bolivia.

Material examinado: PERÚ: Dep. Piura. Prov. Ayabaca: Cerro de Aypate, 2800_2880 m, 14 may 1996, Quipuscoa et al. 629 (F!). Dep. Pasco. Prov. Oxapampa. Oxapampa: Along old road Oxapampa-Montane forest, partly disturbed., 2000 m, 25 jun 2003, van der Werff, Vásquez, Gray, Ortiz \& Dávila 17771 (MO, USM, Herbario Selva Central); Oxapampa: Río el Chuqui, ca. $50 \mathrm{Km}$ of Oxapampa, 1600 m, 27 mar 82, Smith, Pretel \& Palomino 1742 (MO). Dep. San Martín. Prov. Mariscal Cáceres, P. N. Río Abiseo, León 4053 (USM).

\section{Elaphoglossum molle (Sodiro) C. Chr.}

\section{Index Filic., Suppl. 1, 42. 1913.}

Acrostichum molle Sodiro, Anales Univ. Centr. Ecuador 22(161): 171. 1908. Tipo: Ecuador: Napo: Oyacachi, Sodiro s.n., Jan 1900 (Isotipo: S, US)

Se diferencia de Elaphoglossum rimbachii (Sodiro) H. Christ por tener la lámina más ancha $(3,8-4,1$ versus $0,6-2,2 \mathrm{~cm})$ las escamas del envés más separadas entre sí con los cilios ligeramente más largos.

Distribución: Anteriormente conocida de Ecuador y Bolivia, se extiende su distribución al Perú en los departamentos de San Martín y Pasco.

Material examinado: PERÚ: Dep. San Martín. Prov. Rioja. Along road Rioja-Yorongos-La Florida, 1000 m, 29 mar 2001, van der Werff, Vásquez, Gray \& Campos 16473 (USM!, MO). Dep. Pasco. Prov. Oxapampa. Huancabamba: Sector Quebrada Yanachaga, P. N. Yanachaga-Chemillén., 2100 m, 16 set 2004, Mellado 1760 (USM, MO, Herbario Selva Central, HUT,
AMAZ); 2220 m, 17 oct 2004, Mellado, Perea, Becerra \& Mateo, 1989 (USM, MO); 2410 m, 20 set 2004, Mellado \& Becerra 1849(USM, MO, Herbario Selva Central); Lanturachi: Zona de Amortiguamiento del P. N. Yanachaga Chemillén, sector Santa Bárbara, Milpo, 2972-3008 m, 01 feb 2005, Mellado \& Ortiz 2757 (USM, MO, Herbario Selva Central); Oxapampa: Parque Nacional Yanachaga Chemillén, Sector San Alberto, 2200 m, 20 ene 2003, Vásquez, Rojas, Monteagudo, Ortiz, Mateo 27847 (USM, MO, Herbario Selva Central); 2400 m, 21 ago 2004, Mellado \& Francis 1545 (USM, MO, Herbario Selva Central, HUT, AMAZ, HUSA); 1556 (USM, MO, Herbario Selva Central, HUT, AMAZ, HUSA); 1566 (USM, MO, Herbario Selva Central); Oxapampa: Parque Nacional Yanachaga-Chemillén, Sector Chacos. Bosque remanente, borde de carretera, $2219 \mathrm{~m}$, 21 ene 2004, Vásquez, Monteagudo, Peña, Francis 28848 (USM, MO, Herbario Selva Central, HUT, AMAZ).

\section{Agradecimientos}

Nuestros agradecimientos al Jefe y personal del Herbario San Marcos (USM) por las facilidades para la revisión de las muestras botánicas. Franco Mellado agradece al Fondo Christensen, que a través del Jardín Botánico de Missouri, auspició del proyecto para el estudio de la taxonomía y ecología del género Elaphoglossum en el P. N. Yanachaga Chemillén.

\section{Literatura citada}

Kessler, M., R. C. Moran \& J. T. Mickel. 2004. Notes on Bolivian Elaphoglossum. Manuscrito inédito.

Mickel, J. T. 1980. Relationships of the Dissected Elaphoglossoid Ferns. Brittonia, 32(2):109-117.

1985. The Proliferous Species of Elaphoglossum (Elaphoglossaceae) and their Relatives. Brittonia, 37(3):261278.

1990. Two New Species of Elaphoglossum (Elaphoglossaceae) from Amazonas, Venezuela. Annals of the Missouri Botanical Garden, 77:594-595.

. 1991. Elaphoglossum and Peltapteris. En: Tryon, R. M. \& R. G. Stolze (Eds.), Pteridophyta of Peru. Part IV. 17. Dryopteridaceae (Vol. 27, pp. 111-170): Fieldiana, Bot., n.s.

Mickel, J. T. \& L. Atehortúa. 1980. Subdivision of the Genus Elaphoglossum. American fern Journal, 70(2):47-68.

Moran, R. C. 1995. The Importance of Mountains to Pteridophytes, with Emphasis on Neotropical Montane Forests. En: Churchill, S. (Ed.), Biodiversity and Conservation of Neotropical Montane Forests (pp. 359-363): The New York Botanical Garden.

Rojas Alvarado, A. F. 2002. New Species, New Combinations and New Distributions Species of Elaphoglossum (Lomariopsidaceae). Rev. Biol. Trop., 50(3-4):969-1006.

Skog, J. E., J. T. Mickel, R. C. Moran, M. Volovsek \& E. A. Zimmer. 2004. Molecular Studies of Representative Species in the Fern Genus Elaphoglossum (Dryopteridaceae) Based on cpDNA Sequences rbcl, trnL-F, and rps4-TRNS. Int. J. Plant Sci., 165(6):1063-1075.

Smith, A. R., B. León, H. Tuomisto, H. van der Werff, R. C. Moran, L. Marcus \& M. Kessler. 2005. New Records of Pteridophytes for the Flora of Peru. Sida, 21(4):2321-2342. 
